\title{
Cellular Contents and Erythrophagocytic Activity of Buffalo Hemal Node
}

\author{
Jaideep Kaur, Opinder Singh* and Devendra Pathak
}

Guru Angad Dev Veterinary and Animal Sciences University Ludhiana, India

*Corresponding author

\section{Keywords \\ Buffalo, Hemal nodes, \\ Erythrophagocytosis, Erythopoiesis \\ Article Info \\ Accepted: \\ 15 December 2018 \\ Available Online: \\ 10 January 2019}

\begin{abstract}
A B S T R A C T
The present study was conducted on the buffalo hemal nodes $(n=12)$ of different age groups. The cellular elements present in the hemal node were lymphocytes, erythrocytes, neutrophils, monocytes, macrophages, plasma cells, reticular cells and megakaryocytes. The study revealed the presence of hemosiderin pigment formed by the degradation of erythrocytes in the sub-capsular sinus and parenchyma of the hemal node. Macrophages engulfing erythrocytes were observed in sinuses. The amount of the hemosiderin pigment produced was comparatively less in young animals. In calves, few granules of hemosiderin pigment were observed in the sub-capsular sinus as well as in cytoplasm of macrophages and parenchyma of the node, whereas in adult animal's amount of pigment increased indicating the active process of degeneration of erythrocytes by phagocytic cells. Large quantities of hemosiderin pigment was localized in cytoplasm of the macrophages of adult buffalo indicating that erythrophagocytosis was more pronounced in adult buffalo. Megakaryocytes of varying sizes and shape were also present in the hemal node indicating involvement in erythropoietic activity. These cells were usually found in the lymphatic tissue. Some of these cells were multinucleated and were extremely large with irregular outline. The results of the present study suggested active involvement of buffalo hemal nodes in the process of erythrophagocytosis and erythropoiesis.
\end{abstract}

\section{Introduction}

Hemal Nodes are lymphoid organs found in various mammals, especially ruminants. In ruminants hemal nodes are located in subcutaneous regions of neck, mesenteric region, thoracic and abdominal aorta (Cerutti et al., 1998). The hemal nodes in bovine appeared to be lymphoid structures in which sinuses filled with erythrocytes are present in place of lymph sinuses and share the morphological and functional characters of spleen and lymph nodes. Contradictory views have been expressed about the functions of hemal nodes and general biological significance (Ceruttiand Guerrero, 2001). In recent years, some new concepts have been proposed for the function of hemal nodes including erythrophagocytosis, erythropoiesis, platelet formation and immune cell activation (Ceruttiand Guerrero, 2008). An understanding and confirmation of these is essential to establish the possible role of hemal nodes in buffalo. 


\section{Materials and Methods}

The present study was conducted on hemal nodes of buffalo of different age groups. The animals were divided into two groups viz; group 1 including calves up to six months of age $(n=6)$ and group 2 adult animals from three to seven years of age.

The nodes from mesenteric region and along the large blood vessels were collected from animals slaughtered at different abattoirs. Immediately after collection tissue samples were fixed in ten per cent neutral buffered formalin (NBF) to study the histomorphology of hemal nodes.

After fixation, the tissue samples were processed for paraffin blocks preparation by acetone-benzene schedule (Luna 1968) and sections of $5 \mu \mathrm{m}$ thickness were obtained on glass slides with rotary microtome. The paraffin sections were stained with hematoxylin and eosin for routine morphology and Perl's Prussion Blue stain (Sheehan and Hrapchak, 1973) to observe erythrophagocytosis and erythropoiesis.

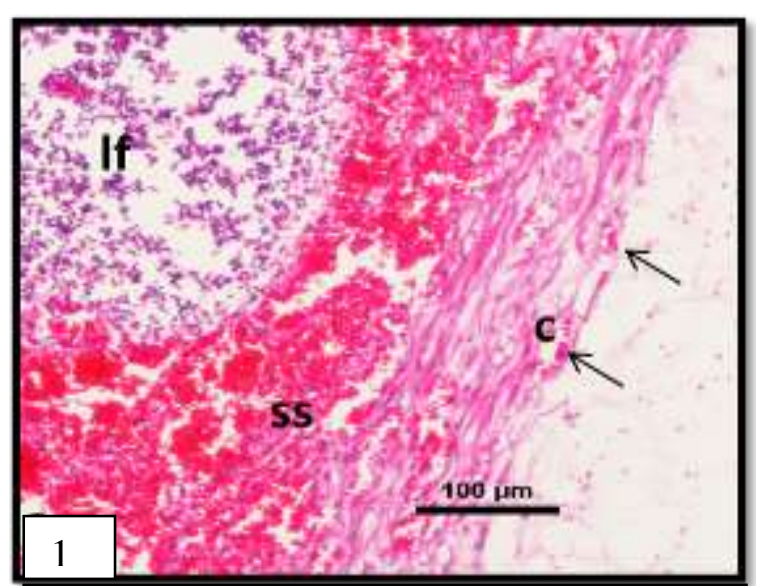

Fig.1 Section of the hemal node of adult buffalo showing the connective tissue capsule (c), subcapsular sinus (ss) and lymphoid follicle (lf). Randomly distributed erythrocytes in capsule were also visible (arrow). Haematoxylin and Eosin X 100.

\section{Results and Discussion}

The hemal nodes were surrounded by connective tissue capsule. Few connective tissue trabeculae extended from the capsule into the parenchyma.

Beneath the capsule, a wide subcapsular sinus was present which was filled with erythrocytes and few lymphocytes were also observed (Fig. 1). Connective tissue trabeculae from the capsule surrounded the trabecular sinuses which remain filled with erythrocytes and were lined by endothelial cells. The parenchyma of hemal node was divided into outer cortex, paracortex and medulla (Fig. 2).

Lymphoid aggregates were observed in the cortex regions which were irregularly arranged in calves and in follicular form in adult buffaloes (Fig. 4). Diffused lymphocytes were observed in paracortex region (Fig. 2). Germinal centers were observed in majority of lymphoid follicles (Fig. 4). Medulla consisted of medullary sinuses filled with erythrocytes and irregularly arranged lymphatic cords (Kaur et al., 2017).

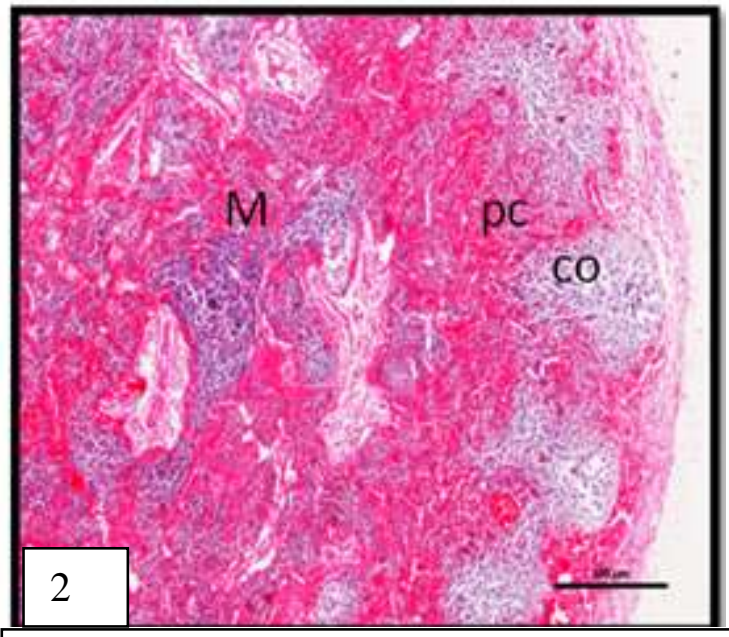

Fig.2 Section of thehemal node of adult buffalo showing the cortex (co), paracortex (pc) and medulla $(\mathrm{M})$ of the hemal node. Haematoxylin and Eosin X 40. 


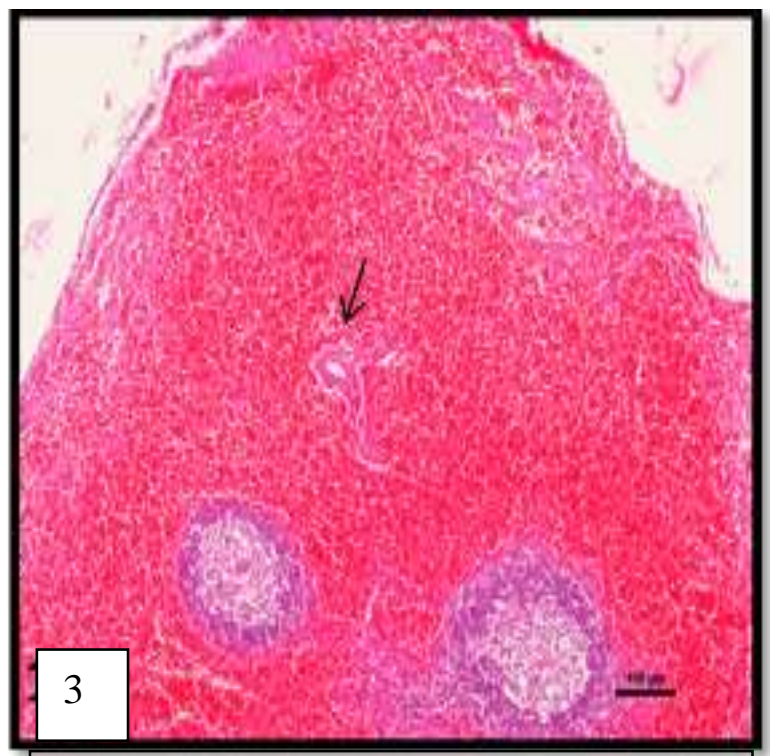

Fig.3 Section of hemal node of adult buffalo completely filled with erythrocytes. Haematoxylin and Eosin X 40.

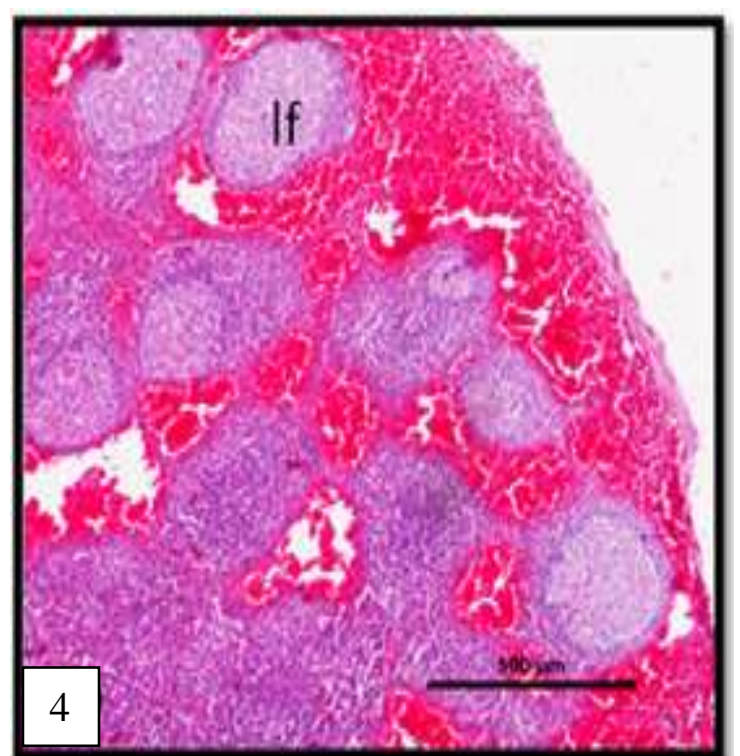

Fig.4 Section of the hemal node of adult buffalo showing the follicular arrangement of lymphoid tissue (lf). Haematoxylin and Eosin X 40 .

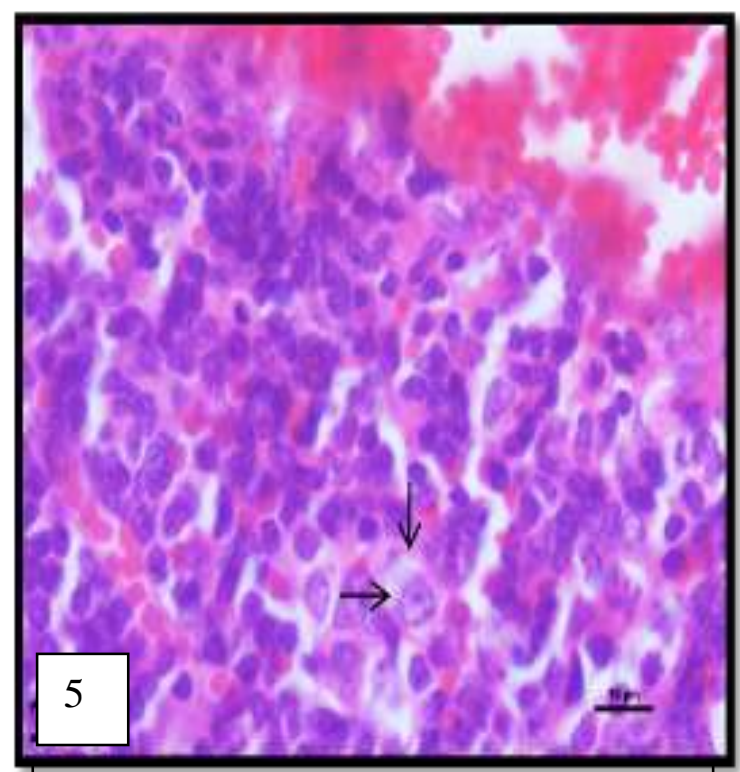

Fig.5 Section of the hemal node of adult buffalo showing the presence of plasma cell (arrow) in the sinus. Haematoxylin and Eosin X 1000.

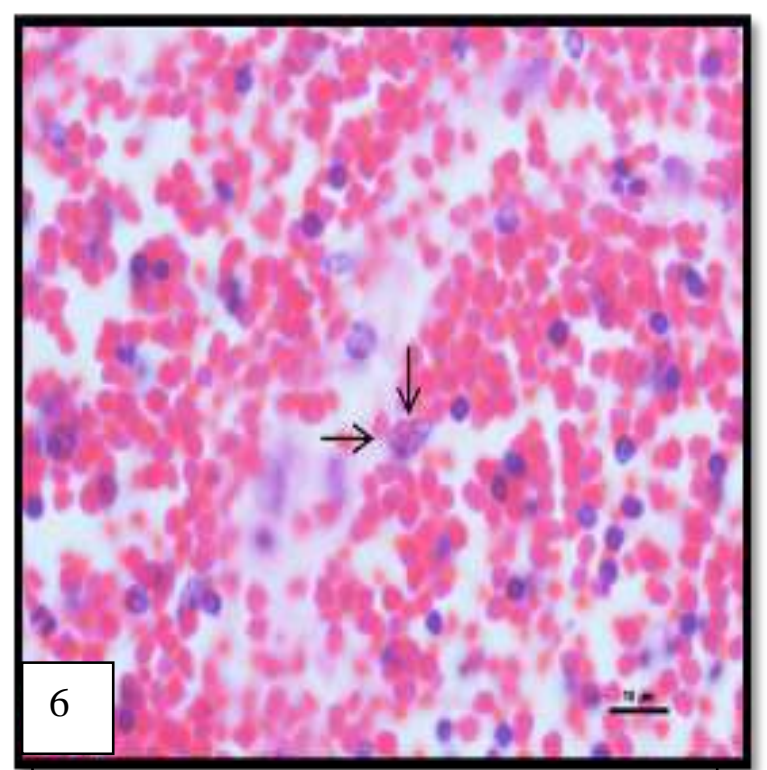

Fig.6 Hemal node showing macrophages (arrow) engulfing erythrocyte in sinuses of hemal node of adult buffalo. Haematoxylin and Eosin X 1000. 


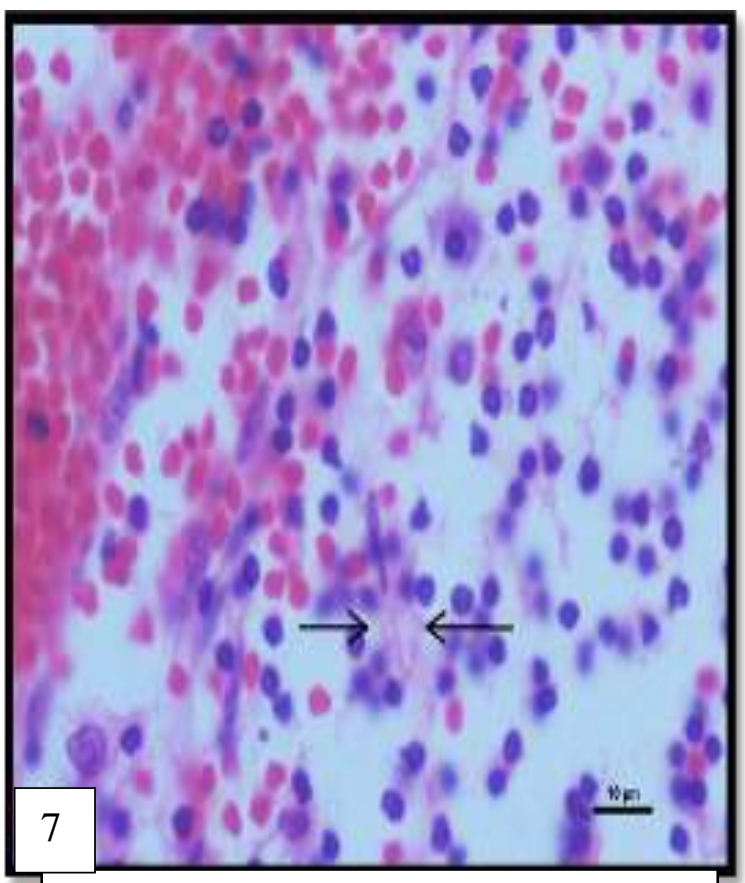

Fig.7 Section of the hemal node of adult buffalo showing the presence of reticular cells in the hemal node (arrow). Haematoxylin and Eosin X 1000.

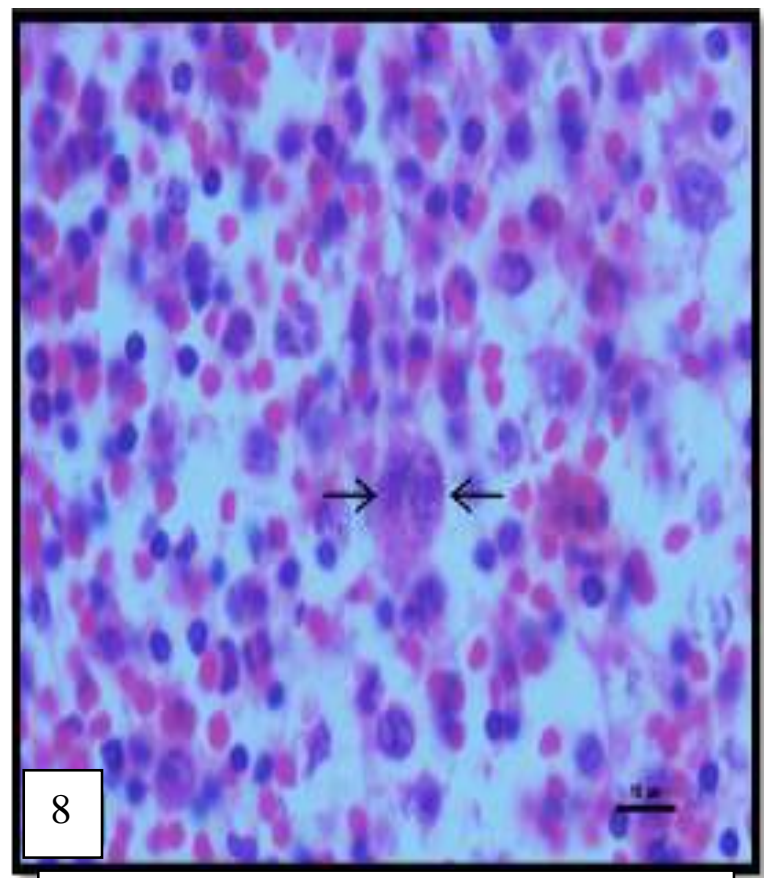

Fig.8 Hemal node section showing the presence of megakaryocyte (arrow) in hemal node of adult buffalo. Haematoxylin and Eosin X 1000.

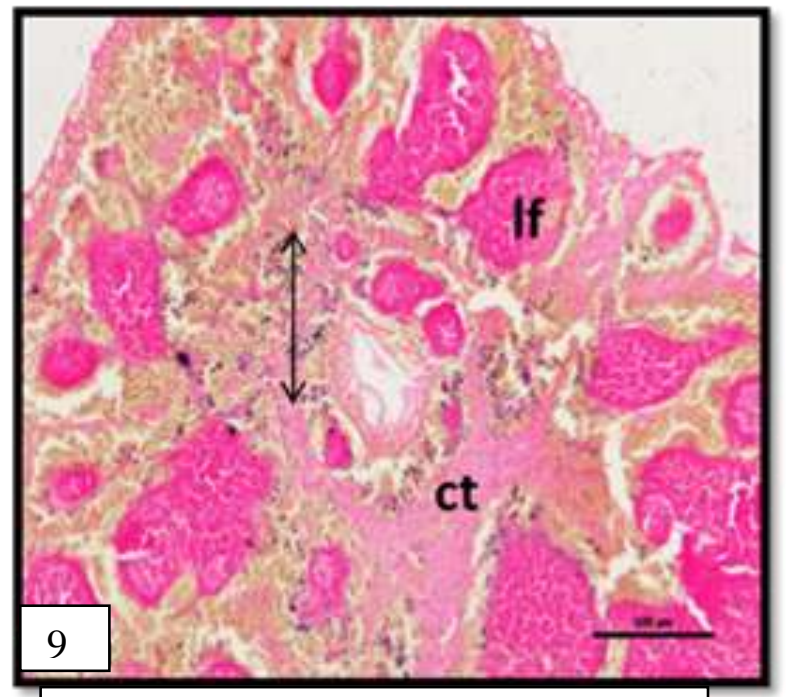

Fig.9 Section of hemal node showing lymphoid follicles (lf), connective tissue (ct) and accumulation of bluish granules of hemosiderin pigment in sinuses (arrow) in adult buffalo. Perl's Prussian blue X 40.

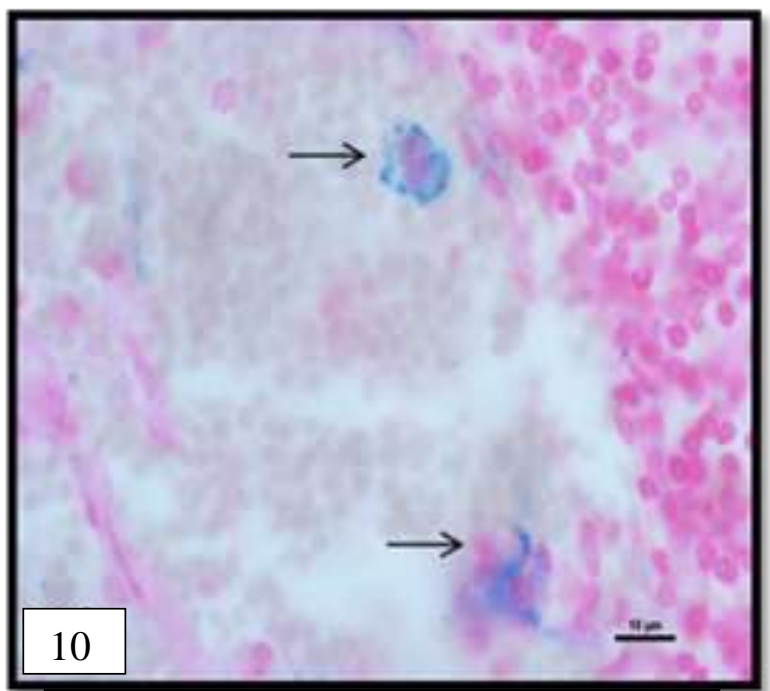

Fig.10 Section of the hemal node showing lymphoid follicle (lf) and accumulation of the hemosiderin pigment in sinus (arrow) of adult buffalo. Perl's Prussian Blue X 100 


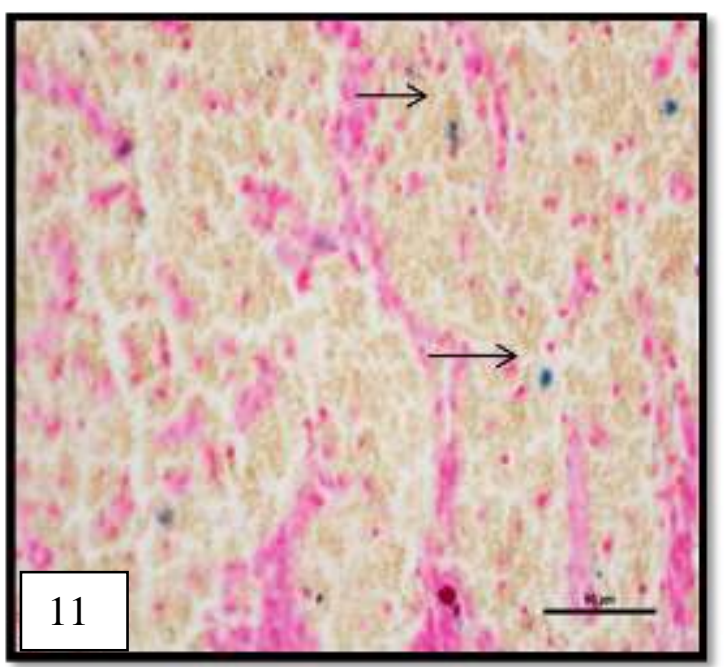

Fig.11 Section of the hemal node showing the accumulation of hemosiderin pigment (arrows) in buffalo calf. Perl's Prussian Blue X 400.
The histomorphological features of hemal nodes showed variations. Some hemal nodes appeared as a sac like structure filled with erythrocytes and few secondary lymphoid follicles with clear germinal centers (Fig. 3). The maximum area of the node was occupied by blood cells with presence of blood vessels in certain areas. The other structural variation which was observed in hemal nodes was presence of red blood cells throughout the parenchyma along with few scattered lymphocytes and empty spaces. No lymphoid mass was found in such nodes.

In majority of hemal nodes, erythrocytes and lymphocytes were found intermingled while in some hemal node they were segregated. In few hemal nodes which contained remnants of lymphatic tissue, the lymphocytes formed small circumscribed areas. There were variations in the size of the lymphocytes within the same or in different nodes. Moreover, the nuclei of the cells present at the periphery of the follicle were usually more regular in shape and often entirely filled with granules while those near the center were

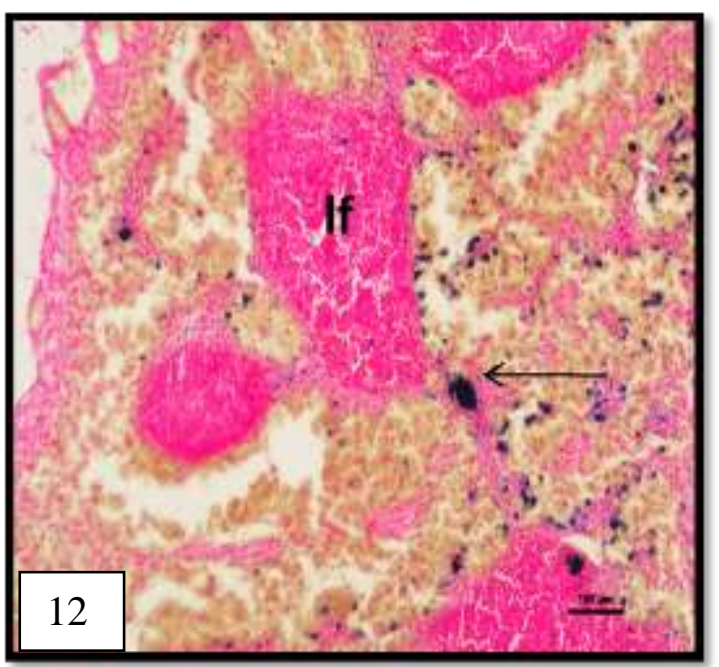

Fig.12 Section of the hemal node showing lymphoid follicle (lf) and accumulation of the hemosiderin pigment in sinus (arrow) of adult buffalo. Perl's Prussian Blue X 100.

vesicular, large and more irregular in shape containing few granules. Polymorphonuclear leucocytes especially neutrophils were commonly observed in parenchyma. They were not common in blood islands and were not observed in the follicles. The cellular content of blood islands at periphery was comprised of numerous lymphocytes and few neutrophils. However, in some hemal nodes in which blood has extensively displaced the lymphatic tissue only few lymphocytes were found among solidly packed erythrocytes. Monocytes were also observed in the lymphatic tissue. Phagocytic cells i.e. macrophages were also present in the subcapsular sinus and lymphoid aggregates of hemal node (Fig. 6). Zhang (2013) reported that except for few erythrocytes, macrophages, reticular cells, and lymphocytes were observed in the sub-capsular sinus and interior blood sinuses of the hemal node. In the cortex, few macrophages and plasma cells were observed (Fig. 5). Reticular cells were also observed in the parenchyma of the hemal node (Fig. 7). Derbalah and Zaghloul (2016) described lymphocytes and lymphoid follicles 
as the main components of the cortex in Egyptian cattle while in the medulla there were wide medullary sinuses, diffused lymphocytes and few lymphoid nodules. The cellular components of the hemal nodes were lymphocytes, erythrocytes, plasma cells, macrophages, mast cells, reticular cells, megakaryocytes and endothelial cells lining the blood vessels.

\section{Erythrophagocytosis}

In the present study the hemosiderin pigment formed by the degradation process was found in the sub-capsular sinus and parenchyma of the hemal node (Fig. 9). Macrophages engulfing the erythrocytes were observed in the sinuses (Fig. 10). These ingested erythrocytes undergo lysosomal degradation and lead to formation of hemosiderin pigment. Choudhary et al., (2011) observed large quantities of hemosiderin pigment in goats produced by degradation of haemoglobin, in both the sinuses and the cytoplasm of some macrophages. The presence of hemosiderin indicated the process of erythrophagocytosis in hemal nodes of goats. However, it was noticed in the present study that the amount of the pigment produced was comparatively less in young animals. In calves, few bluish granules were observed in the sub-capsular sinus as well as in cytoplasm of macrophage and parenchyma of the node (Fig. 11), whereas in adult animals marked reaction was observed (Fig. 12). This indicated the active process of degeneration of erythrocytes by phagocytic cells, resulting into formation of hemosiderin pigment. Large quantities of hemosiderin pigment was observed in cytoplasm of the macrophage (Fig. 10) of adult buffalo indicating that erythrophagocytosis was more pronounced in adult buffalo. Similar observations were made by Cerutti and Guerrero (2008) in buffaloes. They observed large quantities of hemosiderin pigment in both sinuses and cytoplasm of some macrophages of buffalo and transmission electron microscopy images showed numerous macrophages and polymorphonuclear cells with erythrocyte debris in their cytoplasm. Derbalah and Zaghloul (2014) observed that macrophages present in the sinuses of hemal nodes were involved in erythrophagocytosisin Egyptian water buffaloes and this was clearly indicated by presence of the bright blue pigment in some cells. Udoumoh and Ezeasor (2015) reported hemosiderin pigment in sub-capsular sinus, trabeculae and medullary sinuses of adult pigs.

Mega-karyocytes of varying sizes and shape were also present in the hemal node (Fig. 8) indicating involvement in erythropoietic activity. Some of these cells were multinucleated and were extremely large with irregular outline. These cells were usually found in the lymphatic tissue surrounded by more or less completely an empty space.

\section{References}

Derbalah, A. E., and Zaghloul, D.M. 2014. Hemal node of Egyptian water buffalos (Bos bubalus). Journal of Veterinary Anatomy, 7: 79-88.

Derbalah, A.E., and Zaghloul, D. M.2016. Cellular components of hemal node of Egyptian cattle. International Journal of Veterinary and Animal Sciences, 10: 2227.

Cerutti, P., and Guerrero, F. 2001. Identification of positive cells to interleukin-4 in bovine haemal nodes. Anatomia Histologia Embryologia, 30: 219-223.

Cerutti, P., and Guerrero, F. 2008. Erythropoiesis and erythrophagocytosis in bovine haemal nodes. International Journal of Morphology, 26: 557-562.

Cerutti. P., Marcaccini. A., and Guerrero, F. 
1998. A scanning and immune histochemical study in bovine haemal node. Anatomia Histologia Embryologia 27: 387-392.

Choudhary, R. K., Das, P., and Ghosh, R. K. 2011. Post natal development of caprine haemal nodes: a gross and histological study. Journal of Cell and Tissue Research, 11: 2919-2923.

Luna, L.G. 1968. Manual of Histologic Staining: Methods of the Armed Forces Institute of Pathology. 3rd Edn, McGraw Hill Book Co, New York.

Sheehan, D. C., and Hrapchak, B. B. 1973.
Theory and Practice of Histochemistry. pp 80-172.The C V Mosby Co, Saint Louis.

Udoumoh, A. F., and Ezeasor, D. N. 2015. Developmental features of porcine hemal nodes: a histological perspective. Animal Research International, 12: 2241-2248.

Zhang, W. 2013. Studies on the hemal node in Japanese black cattle. Ph.D. Dissretation. The United Graduate School of Veterinary Sciences, Yamaguchi University, Japan

\section{How to cite this article:}

Jaideep Kaur, Opinder Singh and Devendra Pathak. 2019. Cellular Contents and Erythrophagocytic Activity of BuffaloHemal Node. Int.J.Curr.Microbiol.App.Sci. 8(01): 22332239. doi: https://doi.org/10.20546/ijcmas.2019.801.233 